\title{
DORA D'ISTRIA Y LOS GITANOS RUMANOS ${ }^{1}$
}

\author{
Leonardo Piasere \\ Universidad de Verona
}

\section{Resumen.}

Autores de diversas áreas, especialmente del sector de estudios sobre la mujer, recientemente han redescubierto la obra de Dora d'Istria, alias Elena Ghica, una erudita rumana del siglo XIX que escribió considerables informes comparativos sobre las condiciones de la mujer en Europa Occidental y Oriental. También fue la primera mujer en la historia en hablar de las condiciones de las mujeres Gitanas y este artículo trata de analizar la forma en que estas mujeres fueron descritas. En particular, se reconstruye la situación histórico-política de Valaquia en la primera mitad del siglo XIX, ubicando a Elena en la genealogía de los príncipes Ghica, una familia noble del principado, y reexamina la situación de esclavitud a la cual los gitanos en la región fueron sometidos. Por tanto, el artículo analiza la situación personal y contradictoria de Dora d'Istria, una lideresa por la emancipación femenina, quien describió a los Gitanos con dulzura y expurgó la existencia de mujeres Gitanas esclavas con las cuales habría tenido contacto en su juventud.

\section{Palabras clave.}

Gitanos, Valaquia, esclavitud, mujeres.

1. Una version ampliada de este artículo fue el tema de la comunicación «Karl Marx, Dora d'Istria y gitanos rumanos», que leí en el Instituto de Etnología mediterránea, europea y comparativa en Aix-enProvence el 9 de octubre 2013, como parte de mis actividades como investigador invitado en el Labex Med (Laboratorio de excelencia en la Maison Méditerranéenne des Sciences de l'Homme). Doy las gracias de nuevo a Marc Bordigoni, Dionigi Albera y Brigitte Marin por el apoyo recibido durante mi estancia en Provenza. [Traducción del original de David Lagunas]. 


\begin{abstract}
.
Authors from various areas, especially the women's studies sector, have recently been rediscovering the work of Dora d'Istria, alias Elena Ghica, a nineteenth century Rumanian scholar who wrote considerable comparative accounts of the conditions of woman in Western and Eastern Europe. She was also the first woman in history to speak about the conditions of Gypsy women and this article attempts to analyse the way in which these women were described. In particular, it reconstructs the historical-political situation of Wallachia in the first half of the nineteenth century, placing Elena in the genealogy of the Ghica princes, a noble family within the principality, and re-discusses the slavery situation that Gypsies in the region were then subject to. The article therefore analyses the personal and contradictory situation of Dora d'Istria who, a champion for female emancipation, described the Gypsies sweetly and expurgated the existence of female Gypsy slaves with whom she would have had contact in her youth.
\end{abstract}

\title{
Key words.
}

Gypsies, Wallachia, slavery, women.

Recientemente, un artículo se encuentra en proceso de adquirir su pleno valor: se trata de aquel que el gran historiador de la literatura, Fernand Baldensperger, había dedicado en 1938 a lo que denominó "La entrada patética de los Gitanos en las letras occidentales". Siguiendo la huella de Baldensperger, Asseo (2008) y Coquio (2011) muestran que entre los siglos XVIII y XX, grandes escritores y filósofos de Europa continuaron relegando a los Gitanos a los márgenes del horizonte de la civilización europea, aunque su presencia ha sido atestiguada después de los siglos en el seno de las otras poblaciones del continente.

Quiero continuar esta discusión a partir de aquí y hablar de lo que podemos definir como "la entrada patética de los Gitanos en los relatos de emancipación de la mujer en Europa”. En particular, voy a tomar en consideración las obras de Dora d'Istria, una de las iniciadoras de estos discursos. Aunque hoy en día es menos conocida, en el siglo XIX gozó de un gran renombre a nivel mundial. A pesar de conocerla bastante bien, nunca se refirió en sus publicaciones a la esclavitud de los Gitanos habida cuenta de lo que ocurría en ese momento en los principados de Valaquia y Moldavia: las mujeres rom ingresan en los discursos emancipadores de las mujeres a través de esta censura patética.

En el siglo XVIII, varios autores ya habían dado a conocer en Occidente la situación de esclavitud de los gitanos en los Principados. Podríamos citar libros en italiano, en alemán, en francés, que describen de manera más o menos detallada la situación de los esclavos, y que fueron escritos por diplomáticos, académicos, viajeros, aventureros, y también por gobernantes locales como Dimitrie Cantemir, príncipe de Moldavia en 1693 y en 1710-1711, quien escribió en latín el célebre Descriptio Moldaviae de 1714, 
publicado en alemán en 1771. A través de esta obra, descubrimos que no existe ningún noble en el país que no sea propietario de varias familias de Gitanos.

En estos tiempos las buenas relaciones entre los otomanos y Francia permiten a los Príncipes Fanariotas (Griegos de Estambul nombrados por los sultanes otomanos desde 1715 como gobernantes de los dos principados) estar rodeados de intelectuales franceses quienes ocupan las funciones de secretarios, tutores, médicos, enseñantes. Por otra parte, el afrancesamiento cultural de las élites locales de la época se desarrollará de forma paralela enviando a los jóvenes a estudiar a Francia, en un contexto general en que el francés era la lengua de la diplomacia y del cosmopolitismo nobiliario. Es así precisamente como en los años cuarenta del siglo XIX la Societatea Studenţilor Români (Sociedad de los estudiantes rumanos) ve la luz en París. En las primeras décadas del siglo XIX, una especie de estela transnacional se forma y une a Iaşi y Bucarest a París, con los Moldo-Valacos afrancesados y los Franceses rumanófilos, al interior de la cual se produce un intercambio y un flujo continuo de ideas, información, viajes, y a veces conspiraciones. Esta estela se desarrollará a lo largo del siglo, tanto es así que George Bengesco, en 1895, reunirá 952 obras escritas en francés, por los franceses o los rumanos, referidas a Rumania. Se comprende entonces el porqué, en la primera mitad del siglo XIX, son primeramente los autores liberales franceses rumanófilos y los autores rumanos afrancesados los que ejercen de portavoces a nivel europeo de la situación de los Gitanos esclavos. Al mismo tiempo que el movimiento contra la esclavitud se desarrolla en su conjunto, especialmente en Inglaterra y Francia, y surgen las sociedades contra la trata y la emancipación de todos los esclavos en las colonias, la presencia de la esclavitud en los países cristianos y europeos como Valaquia y Moldavia comienza a parecer cada vez más insoportable a los ojos de las potencias occidentales de la época. Paulatinamente, la liberación de la dominación otomana -sin caer bajo la dominación rusa o de los Habsburgo- y la unificación política de los dos principados avanzan a la par en las informaciones de Occidente de la primera mitad del siglo XIX, de manera coincidente con la denuncia sobre la condición de los campesinos víctimas de abusos por parte de los boyardos (nobles locales) y la esclavitud de los Gitanos. Es muy raro encontrar un libro sobre los principados de la época que no se refiera indistintamente a su variada composición étnica y a la presencia de la esclavitud gitana. Georges Bengesco hace hincapié en que los primeros libros sobre los principados escritos por autores locales aparecen en 1837. Es significativo que dos de estos libros sean de Mihail Kogălniceanu (Kogalnitchan 1837a, 1837b), quien se convertirá en uno de los fundadores de la actual Rumania, y que se abordeen uno la historia de los dos principados, y en el otro la situación de los Gitanos! Este segundo libro contribuirá a la divulgación definitiva, en Europa, de la situación de la esclavitud gitana: la independencia rumana y la esclavitud gitana estarán siempre asociadas. 
Cuando Kogălniceanu escribe, nos encontramos en un período particular de la lucha histórica en los principados, que tan bien describirá Henry H. Stahl (1969), entre los campesinos y los señores territoriales. La servidumbre de la gleba había sido instaurada oficialmente en el siglo XVII, fijando al campesino a una propiedad, pero en el siglo XVIII, con la entrada gradual de los Principados en el comercio internacional de trigo, la servidumbre de la gleba es abolida oficialmente y reemplazada por el sistema de corbeas: los campesinos poseen la tierra que pueden trabajar por sí mismos, pero también deben trabajar para el terrateniente (al que también tienen que dar el diezmo). A cambio, los boyardos obtendrán la formalización de la esclavitud gitana. En 1831, después de la enésima invasión de los Principados por Rusia, el Reglamento orgánico (Reglement organic) fue adoptado: se trataba de un conjunto de leyes, que con el pretexto de la modernización de los dos principados, decretaba la victoria de los boyardos sobre los campesinos y garantizaba al mismo tiempo la soberanía de Turquía sobre los principados y la "protección" de Rusia. Sin embargo, el Reglamento orgánico mantiene la esclavitud de los Gitanos. Se centra, en particular, en los esclavos del Estado, puesto que determina los impuestos que deben pagar, y manifiesta un interés particular en la sedentarización de los nómadas, convirtiéndolos en granjeros corbeables (Regulamentele organiceale Valahieişi Moldovei, 1944: 257-260; Achim, 1994: 90-91; 2004). Los Gitanos eran distribuidos desde hacía mucho tiempo en tres categorías de amos: el Estado, el clero y los boyardos. En el trascurso de los siglos, los príncipes habían dilapidado abundantemente la reserva de Gitanos del Estado por medio de fuertes donaciones unas veces a los monasterios, otras a los boyardos (Piasere, 2012), pero los Gitanos del Estado permanecieron en un número considerable: ahora que los boyardos tenían más necesidades de mano de obra para sus grandes propiedades, el Reglamento orgánico les permitía tratar de convertirlos en granjeros corbeables, sobre todo los nómadas, y mantener los que ya poseían -nómadas o sedentarios- en un estado de esclavitud.

Por ejemplo, en Valaquia, de los 2,5 millones de habitantes, 2 millones eran campesinos, de los cuales el $25 \%$ vivía en los pueblos libres y el $75 \%$ en los pueblos sometidos a las corbeas en las tierras de los boyardos. Estos a su vez representaban una pequeña minoría, en torno a 15.500 personas, pero sus propiedades feudales representaban casi el 80\% del área rural del país (Stan, 1971: 14-15). Los esclavos Gitanos representaban alrededor del 7\% de la población. A pesar de haber sufrido varios cambios a lo largo de los siglos, y a veces variaciones en el nombre (Mircea, 1951), la robie, como se designaba en rumano a la esclavitud gitana, podría ser catalogada como una forma intermedia entre la "esclavitud total" y la "esclavitud atenuada", de acuerdo con la tipología de Moulier Boutang (2002: 646), pero más desplazada hacia este segundo extremo. De acuerdo con los códigos civiles promulgados a principios del siglo XIX, del lado de la esclavitud total encontramos la alienabilidad (incluyendo la separación de los miembros de la familia), la prohibición de casarse con los no-esclavos, la condición de los niños (que eran esclavos 
o libres de acuerdo con la situación jurídica de la madre, no del padre), la prohibición de la posesión de la tierra, los casos raros de liberación. Del lado de la esclavitud atenuada, nos topamos con la cuestión quizás más importante de la existencia de ciertos derechos, como el derecho a la vida (en teoría, el amo no tenía el derecho de vida y de muerte sobre los esclavos), el derecho parcial a la propiedad (propiedad personal, no la tierra) y lo más importante, la posibilidad de vender los productos que los Gitanos fabricaban en calidad de artesanos.

Remito a otros estudios recientes en lo que respecta a otras características de la situación de los esclavos gitanos moldo-valacos (Achim, 1998; Petcuţ, 2007; Piasere, 2012: 81121), pero este contexto es el que organiza nuestro punto de partida para leer las páginas de Dora d'Istria. Debemos por tanto retomar ciertos hechos que tuvieron lugar entre 1828 (año de la enésima guerra ruso-turca) y 1848 en Bucarest, en aquel tiempo la capital de Valaquia. La ciudad entonces gozaba de una situación cultural híbrida, con multitud de intelectuales franceses establecidos como profesores o preceptores de la nobleza local, con jóvenes nobles que viajaban entre Francia y Valaquia, con los gobernantes, que aun cuando alimentaban secretamente ideas independentistas y nacionalistas, debían hacer acrobacias políticas entre Estambul y Moscú, y en el interior, afrontar los embates del ala liberal de boyardos y del ala conservadora compuesta de grandes terratenientes. En el seno de estas familias de boyardos, existía a menudo una división entre los liberales nacionalistas y los conservadores rusófilos y/o turcófilos.

Entre las figuras de esta presencia franco-valaca que cobra importancia en esta situación social, Jean Alexandre Vaillant es quien nos interesa. Vaillant está hoy prácticamente ausente en los estudios romológicos, pero su figura debe ser reconsiderada. En 1829 es llamado para enseñar en una escuela prestigiosa de Bucarest, que se consolidaba como un lugar de transmisión de ideas francófilas, nacionalistas y liberales. Al mismo tiempo, sirve como tutor de los hijos de boyardos y príncipes, incluidos los príncipes reinantes de la época, los Ghica. Sin embargo, parece mostrar excesivo celo en su propaganda como francmasón liberal y se inmiscuye demasiado en las relaciones entre familias de boyardos, hasta el punto que en 1834, el príncipe de Valaquia, Alexandru II Ghica, se ve obligado a relevarlo de sus funciones. Permanece en Bucarest, donde publica unos años más tarde una gramática y un diccionario de rumano. Los rusos, dueños de la política local, preocupados por el aumento del nacionalismo que se extiende a través de la valorización de la lengua local, lo consideran un obstáculo en todos los sentidos. Alexandru II Ghica es un príncipe débil, totalmente en manos de su hermano Mihai Ghica, quien ocupa el cargo de mare ban (la segunda función del Estado, que corresponde grosso modo a la de Primer Ministro). Alexandru se ve obligado a hacer juegos de malabares entre los rusos (que lo han escogido) y los turcos (con quienes mantiene un contencioso histórico de orden familiar) y tiene un don para mezclarse una y otra vez 
con los boyardos, sosteniendo a los rusos y a los campesinos, con los rusos, apoyando a los boyardos y los intereses nacionales, o con los liberales radicales, combatiéndolos directamente. Vaillant participa en un complot en 1839 junto con otros intelectuales y jóvenes boyardos afrancesados, y es expulsado definitivamente de los Principados en 1841. De vuelta a París, Vaillant frecuenta los ambientes rumanos de la ciudad y se convierte en miembro de la Société Orientale, una institución, nos dice Nicolae Iorga (1918), que aspiraba en algún momento a desarrollar investigaciones sobre los Gitanos en Rumania, las cuales nunca se llevaron a cabo. En 1844, Vaillant publica los tres volúmenes de Romanie. Partidario de la unificación de todos los pueblos rumanófonos (incluyendo los de Transilvania, Besarabia y Banatde Timisoara), parece que fue él quien designó a todos estos pueblos con el nombre de "Rumania", el cual se impondrá a partir de entonces, cuando en ese momento solo estaba reservado para Valaquia (Iorga, 1918). En 1862 regresó para realizar una estancia corta en Rumania, cuando finalmente los dos Principados se unieron definitivamente, y fue naturalizado como rumano en 1864.

En 1854, Vaillant publica en la l'Illustration (el famoso semanario parisino) una serie de artículos sobre los Gitanos. Estos artículos completarán el libro Les Rômes. Histoirevraie des vraisbohémiens, que se publicará en $1857^{2}$. El libro es ahora poco digerible por todas las etimologías que ya no se sostienen por sí mismas y que el autor se obstina en proponer, y también debido a sus argumentos extravagantes sobre los vínculos de los Roms con los diversos pueblos de la tierra; pero la parte en que narra los eventos autobiográficos y la situación de los Roms en los dos Principados es interesante. Observamos entre otras cosas que, incluso años más tarde, continúa participando en el juego político del principado. Por ejemplo, bendijo a los Ghica como adalides de la emancipación de los Gitanos esclavos en Valaquia, mientras que los príncipes que los habían liberado eran sus enemigos, los hermanos Bibescu, representantes del ala conservadora, como se muestra en la Tabla 1 que resume el proceso de emancipación ${ }^{3}$.

\begin{tabular}{|l|l|l|}
\hline FECHA & EMANCIPACIÓN & PRÍNCIPE/AUTORIDAD \\
\hline VALAQUIA & Gitanos del Estado & GheorgheBibescu \\
\hline 22 marzo 1843 & Gitanos del clero & GheorgheBibescu \\
\hline 11 febrero 1847 & Gitanos de los boyardos (no aplicado) & Gobierno provisional \\
\hline Junio 1848 & Gitanos de los boyardos & BarbuŞtirbei \\
\hline 20 febrero 1856 &
\end{tabular}

2. En 1868, Vaillant publicará también una recopilación sobre la lengua de los Roms rumanos. Es interesante señalar que numerosas frases recogidas en los diálogos (p. 51-89) tienen como objeto el cuidado del cuerpo y de la casa, lo cual nos hace sospechar que el enseñante o los enseñantes de Vaillant fueron vatraşi, los Gitanos esclavos reservados para el trabajo doméstico. Una frase dice: "samrob, ouray ", que Vaillant traduce como "Señor, yo soy su siervo" (p. 55). La palabra rob del diccionario es traducida como "esclavo, paciente, resignado" y el verbo robimpor "yo soporto" (p. 124).

3. A pesar de tener un nombre diferente, Gheorghe Bibescu (o Bibesco) y Barbu Ştirbei eran hermanos. 


\begin{tabular}{|l|l|l|}
\hline FECHA & EMANCIPACIÓN & PRÍNCIPE/AUTORIDAD \\
\hline MOLDAVIA & Gitanos del Estado & MihailSturdza \\
\hline 14 febrero 1844 & Gitanos de los boyardos (no aplicado) & Comité revolucionario \\
\hline Agosto 1848 & Gitanos de los boyardos & Grigore A. Ghica \\
\hline 22 diciembre 1855 &
\end{tabular}

Tabla 1 - Cronología de la liberación oficial de los Gitanos esclavos.

En realidad, en 1838, Alexandru II Ghica no había hecho sino aplicar ciertas disposiciones previstas del Règlement organique, cuyo objetivo era transformar a los Gitanos en campesinos corbeables por medio de complicadísimos mecanismos, combinando modalidades de reembolso y los impuestos a pagar; gracias a una hábil campaña publicitaria en periódicos extranjeros, había hecho creer que estos cambios suponían la emancipación de los esclavos, cuando en verdad no dejó rastro alguno de emancipación. Algunos liberales morderán el anzuelo, como Vaillant, quien aplaudirá a Alexandru II, mientras otros no, como Colson (1839), quienes denunciarán la confusión. El príncipe sucesor, el rusófilo Bibescu (Bibesco, 1894), dispondrá de buenas cartas para demostrar que él estuvo en el origen del proceso de emancipación. Esto es innegable, desde el punto de vista jurídico, pero nos equivocaríamos desde un inicio si tomáramos como ciertas las supuestas aperturas liberales: Adolphe Etienne Billecocq, cónsul de Francia en Bucarest desde 1839 hasta 1846, explica bien que los esclavos del clero, recién liberados, se preparaban para llevar una vida de miseria aún peor que antes, privados como estaban de apoyos sociales. Todo ello, ironizaba, "para otorgar a Hospodar Bibesko la única gloria que le faltaba aún, la de trabajar por el bien de la humanidad." (B. A. 1847:183). La esclavitud gitana se vio afectada directamente por esta pelea hipócrita, es decir, el saber a quién correspondía el mérito de ser el primero en haber emancipado a los Roms...

Si me detengo en los detalles de estos eventos y en las personas, es porque nos ayuda a comprendermejor la posición de nuestro personaje. Así, es bajo el nombre de Dora d'Istria que la princesa rusa Elena Mikhailovna Koltzova-Masalskaya firmaba sus escritos, pero en realidad era el nombre que había adquirido por su matrimonio con el conde Alesandre Koltzoff-Masalski, mientras que su nombre de soltera era Elena Ghica. Y Elena Ghica no es otra que la primogénita de Mihai Ghica, el mare bande Valaquia durante el reinado de Alexandru II Ghica, su hermano, el enemigo de los conspiradores de 1839. Elena había nacido en 1828, cuando reinaba otro hermano de su padre, Grigore IV Ghica. Mihai Ghica es el "Michel Ghika" que censura Vaillant por condenar a tantos liberales y, probablemente, por haber jugado un papel incluso en su expulsión... Sin embargo, Mihai Ghica es también el fundador del Museo Arqueológico en Bucarest, el que ama el clasicismo y la cultura alemana, y el que procuraba tutores extranjeros para sus hijos. Vaillant, según algunas fuentes, fue uno de los tutores de su hija Elena. Vaillant y Mihai Ghica comparten la misma suerte, en el sentido de que han partido de Bucarest 
con un intervalo de pocos meses de diferencia, el primero habiendo sido expulsado y el segundo relevado de sus funciones. Mihai lleva entonces a su familia de viaje por Europa, durante unos años. Así, en Sajonia, Elena, muy estudiosa y muy preparada, logra los favores de Alexander von Humboldt; en Venecia, donde la familia permanece en 1848, goza de la oportunidad de asistir a la revuelta de y Daniele Manin se convierte en uno de sus héroes. Elena es una aristócrata, pero pertenece a una dinastía que durante mucho tiempo ha tratado de anteponer los intereses nacionales frente a los de los imperios de los cuales los Principados dependían; también pertenece a una familia de las menos tradicionalistas de los Ghica, y está evidentemente impregnada de las ideas liberales que Vaillant y compañía pregonaban en Bucarest. En 1849, Elena se encuentra de nuevo en la capital de Valaquia, donde se encuentra con un militar, el conde ruso Koltzoff-Masalski, con quien se casará ese mismo año. Parte con destino a San Petersburgo, donde frecuenta la corte de los zares; pero su matrimonio no va bien, el ambiente político reaccionario en el que se encuentra tampoco le favorece, y después de la enésima invasión de los Rusos en los Principados, expresa públicamente su protesta y recibe por ello varias advertencias. Abandona Rusia y se va a vivir a Suiza, pero nunca se divorciará de su marido, quien no regresará con ella jamás, y mantendrá para siempre la nacionalidad rusa. Cuando el primer libro de Dora d'Istria aparece en 1855, descubrimos a una autora completamente original; alternará la creación literaria con los relatos de viajes, y su renombre mundial crecerá sin cesar, hasta su muerte, que se producirá en 1888 en Florencia, la ciudad donde se había instalado en 1870. Elena Ghica no regresará nunca a Rumania, cuyo recuerdo permanecerá siempre en su seudónimo, Dora d'Istria, el cual no se relaciona con la región de Istria y que parece significar "Nostalgia del Danubio".

Ella ejercerá una actividad frenética como escritora, siempre y únicamente en francés, y le llevará a publicar seis obras solo entre 1855 y 1865, con un total de quince volúmenes, algunos de los cuales fueron rápidamente reimpresos con adiciones o traducidos a otros idiomas. Durante este periodo, comienza a colaborar con revistas importantes en Francia, en Italia, en Rumania, en Estados Unidos, etc.; con asiduidad, los artículos son reeditados, y algunos son recopilados como parte de otros volúmenes autónomos. El primer intento de recopilación bibliográfica de sus obras se remonta a 1868, pero la reconstrucción de su bibliografía se encuentra al día de hoy todavía incompleta. Al igual que fue célebre durante su vida, fue olvidada o casi olvidada durante un siglo. Apenas se le prestó atención en el trascurso del siglo XX en Rumania, y esta falta de interés ha creado un serio compromiso de estudio, pero solo en las últimas décadas, sobre todo por parte de autores rumanos (Bodea, 1998; Bordas, 2005) e italianos (Rossi, 2005: 232253; D’Alessandri, 2007). Experta en tradiciones albanesas, convencida de que este fue el origen de su familia, su herencia es ahora invocada desde los ambientes intelectuales albaneses, provocando el escándalo de los descendientes actuales de los Ghica: la grafía de su nombre es "re-albanizada" como Gijka, en Tirana le dedican calles, fundaciones, 
publicaciones (por ejemplo Sauku-Bruci, 2004). En este tiempo, algunas de sus obras son reeditadas, no solo en Albania, sino también en Serbia, en Grecia, en Turquía; su figura empieza a ser objeto de congresos especiales (como los que tuvieron lugar en Florencia en 2008, en Tirana y Bucarest en 2012) y comienza a ser citada en las revistas de estudios feministas (Dibra, 2006), y a ser objeto de comunicaciones en los congresos de los genderstudies.

Es imposible aquí esbozar las líneas complejas de su pensamiento, pero si seguimos a Antonio Alessandri, en la monografía quizás más completa y más imparcial que lo analiza, podemos resumir los tres centros principales de sus intereses: 1) la dialéctica entre religión y política después de la Revolución Francesa; 2) las asuntos nacionales de las poblaciones balcánicas, especialmente Rumania, Grecia y Albania, pero también Serbia, Bulgaria, Hungría; 3) la condición femenina en Europa occidental y oriental (2007: 13). Muchos otros temas se expresan a través de su pluma, pero en general, también podríamos resumir su pensamiento diciendo que Elena, impregnada de un nacionalismo romántico, se posiciona como una aristócrata liberal favorable a la independencia de muchos "pequeños pueblos" frente a todos los autoritarismos imperiales, de ahí su interés respecto a las nacionalidades "ocultas" de la época como la nacionalidad albanesa, posiblemente también en vías de reivindicaciones reales; fue cristiana pero anticlerical, opuesta a la tiranía de la Iglesia Católica, crítica respecto a la Iglesia ortodoxa atrasada y confinada en los conventos, y atenta a las innovaciones introducidas por la Reforma; convencida de la misión civilizadora de los Europeos, considerando a la civilización cristiana como superior a todas las demás, manifestaba un etnocentrismo radical, sobre todo contra los "asiáticos", y al mismo tiempo una especie de relativismo, inspirador, si alguna vez lo fue, del diálogo entre los Europeos del Este y del Oeste; partidaria convencida del Estado nacional regido por una monarquía constitucional, divisó el progreso en el desarrollo del capitalismo y, por encima de todo, en la implicación de las mujeres en la emancipación nacional. De ahí la importancia constante que Dora d'Istria ha tenido en la educación de las mujeres. Como destaca D’Alessandri, Dora d'Istria siempre se opuso a las teorías socialistas, "ella subrayó la necesidad de garantizar la seguridad femenina y los derechos de los capitalistas de todos los países, y es en este contexto que la princesa confería a las mujeres 'una importante función regenerativa de la sociedad a partir de la idea de que la mujer es la correa de transmisión de los cambios sociales más profundos"'; las mujeres, en opinión de Dora d'Istria, debían trabajar enérgicamente para civilizar al país: así la patria, apreciando sus capacidades, no ofrecería ninguna prueba de ingratitud bajo esta consideración" (2007: 173). Ella postulaba la plena igualdad de derechos entre hombres y mujeres. La regeneración de la humanidad requiere por tanto de la libertad de los países frente a las tiranías y del compromiso que las mujeres sabrán adoptar en el seno de su propio país. Su implicación en este combate fue siempre exclusivamente intelectual, y siempre se mantuvo alejada de "todas las formas de activismo político y radicalismo" 
(2007: 236). Su militancia se manifiesta en el estudio, en el sentido de que sus propuestas derivan siempre de investigaciones documentadas. Es en este punto donde desarrolla un estudio comparativo en profundidad sobre la situación de las mujeres en el seno de los pueblos conceptuados a lo Herder, y su obra puede ser apreciada, en efecto, como la de un Herder femenino. Su presentación es a menudo de factura esencialista hoy poco digerible, pero tiene el mérito de proponer regularmente interpretaciones precisas y de ubicar en la escena intelectual a pueblos sin poder o sin Estado, incluso a las mujeres de pueblos sin poder o sin Estado. En el marco de un anti-orientalismo dominante, no obstante, tiene el valor de exponer un relativismo cultural temerario cuando se dirige a Europa criticando a los autores occidentales por haber retratado a golpe de estereotipos a las mujeres de Europa Oriental, sin conocer en profundidad la historia y las costumbres locales, y sin considerar mínimamente la reflexividad. El concepto de "castidad" no posee el mismo significado en Oriente y en Occidente, señalará por ejemplo. Y en efecto, tiene el mérito de plantear una reflexión sobre la situación de las mujeres en Europa, presentándose como una intérprete entre el Oriente y el Occidente. Sus dos obras más conocidas sobre el tema son Les femmes en Orient, de 1859-60, y Des femmes par une femme, de 1865, donde el contenido es en este caso se refiere a las mujeres de Europa occidental.

Gracias a ella las mujeres gitanas irrumpen en el discurso sobre la condición femenina. Por otra parte, y de acuerdo a mi conocimiento, Dora d'Istria es la primera mujer que escribe sobre los Gitanos, aunque para los simpatizantes de los estudios rom, me parece, sigue siendo una perfecta desconocida: su nombre está ausente de toda memoria bibliográfica sobre los Gitanos que conozco. No obstante es en estas dos obras donde hallamos dos pasajes extensos sobre los Gitanos. En Les femmes en Orient, obra en dos volúmenes cada uno dividido en seis libros, la autora habla de las mujeres de los diferentes pueblos de Europa del Este y de Asia. Los Gitanos, con los Armenios y los Judíos, aparecen al inicio del primer volumen, en el apartado dedicado a Rumania, en un capítulo titulado "Los asiáticos en Rumania" (1859, Vol. I: 103-110). En Des femmes par une femme, las mujeres latinas se ubican en el primer volumen y las germánicas en el segundo. Los Gitanos aparecen en el último capítulo del segundo volumen, en un capítulo titulado "Les Hindouesdans les sociétés germanique et latine" (1865, vol. II: 339-355).

Esta última es una descripción, no siempre lineal, de los Gitanos en algunos países de Europa occidental, la cual se inspira esencialmente en un largo artículo de Alphonse Esquiros, otro anticlerical convencido, publicado en la Revue des Deux Mondes en 1858. El autor, en el exilio en Inglaterra desde 1851 por oponerse al golpe de Estado de Napoleón III, retoma como punto de partida una breve estancia en Hampshire con los Stanley (que se definen a sí mismos en rumano como Barengres), Gypsies de New Forest, para realizar un rastreo de la situación de los gitanos, principalmente con la ayuda de 
textos por aquel entonces célebres de Grellmann, Mérimée y Borrow. La descripción de Dora d'Istria, aunque no ceja en comparar las diferentes ideas de los autores, es un resumen servil que a menudo raya en el plagio: llegará a utilizar la descripción de Esquiros de los Gitanos rusos, músicos y danzantes expertos que, en la lectura, parecían provenir de la experiencia de la autora en San Petersburgo. El marco resultante puede parecer banal a los ojos de hoy en día, y el hecho de que exista un capítulo dedicado a los Gitanos en la estructura de la obra parece efectivamente más importante que el contenido de este capítulo. Aunque parece seguir, de algún modo, la teoría kantiana del racismo de los “instintos convertidos en hereditarios" (1860: 351), según la cual ciertos comportamientos negativos provienen de su origen hindú, o más precisamente de su origen hindú mezclado "con las poblaciones negras davidianas" (p. 344), incluyendo por ejemplo la "suciedad incurable de la cual Asia nunca se ha sonrojado" (1865: 344), hay que decir, sin embargo, que Dora d'Istria, espulgando notablemente las noticias sobre las mujeres, no adolece de un impuso interpretativo original. Para ella son mujeres fieles, contrariamente a sus maridos, y madres tiernas, y se puede afirmar que la bohémienne "es mucho más inteligente que el hombre" (p. 347). Asimismo, realiza el elogio especialmente de su arte de la adivinación y se ríe de "la ignorancia y la credulidad de las clases bajas de ciertos países" (p. 349). Dora d'Istria anticipa aquí las consideraciones que serán desarrolladas por la antropología de los Gitanos cien años más tarde (Okely, 1996: 94114; Ferrari, 2012), haciendo hincapié en que las adivinadoras gitanas saben utilizar su propia cosmología para burlarse y explotar las cosmologías mágico-religiosas locales, y Dora d'Istria identifica en su escepticismo consecuente el "genio astuto" del que están dotadas las "razas asiáticas" (p. 350).

El estudio precedente de 1859 es de mayor interés para nosotros. Ella cavila en la parte reservada a los rumanos, en lo profundo de un elogio hacia su país de origen, el cual habría acogido, a lo largo de los siglos, a los refugiados de diferentes religiones llegados de otras partes, siendo "la única nación latina que no habría torturado a nadie con el pretexto de la religión” (1859: 85). Este es el caso de los Armenios que huían de la ocupación de sus tierras por los persas en el siglo XI, los Judíos españoles expulsados en el siglo XV, los protestantes perseguidos por los Habsburgo en el siglo XVI. Y señala particularmente a Isabel la Católica:

Esta cruel princesa -queriendo justificar su nombre- protege en España este mahometismo cristiano llamado catolicismo. La Inquisición española había adoptado con razón, los colores del profeta, el verde. Nunca el islamismo ha mostrado, en modo alguno, el fanatismo atroz del cual han estado impregnados hasta nuestros días los actuales gobiernos que se han sucedido en España (1859: 84) .

En este país de acogida, se encuentran también Asiáticos inmigrantes, Armenios, Judíos 
y los Gitanos. En lo que concierne a los dos primeros, provenientes del Próximo Oriente, ella plantea descripciones poco banales de sus diásporas. En cuanto a los Gitanos, son "una tribu del sur de Asia" que se ha refugiado, dice, por razones aún desconocidas a orillas del Danubio, en el siglo XV. El lector no debe sorprenderse si ubica a los Gitanos "al margen del cristianismo ", dice la autora, ya que si bien:

es cierto que en Rumania, como en otros lugares, participan de buen grado en las ceremonias del culto dominante, ha sido hasta ahora imposible convertirlos realmente. Las Tsigankas con las cuales he tratado de abordar estas cuestiones, me han parecido siempre tan indiferentes a los temas religiosos como un mandarín chino (p. 108).

Tras esta evocación de sus diálogos con las Gitanas, podría esperarse una descripción de primera mano. Pero, toda la sección es en gran parte una amalgama de datos tomados de nuevo de Esquiros y, en lo que respecta a Rumania, de alguien que conocemos bien: Vaillant. Vaillant fue uno de los tutores de Elena Ghica, y sin embargo nunca se menciona aquí. La supuesta liberación de esclavos de Alessandru II Ghica en 1837, de la cual habla Vaillant, no se incorpora en la obra de Dora d'Istria sobre la historia de la esclavitud, sino que se inscribe en una historia edificante de Rumania y la dinastía de los Ghica. En efecto, la autora lee a su modo los dos modelos que en otro texto (Piasere, 2004) he denominado el enfoque occidental y el enfoque balcánico en las relaciones Gitanos/noGitanos. Mientras que en Occidente los Gitanos han sido perseguidos, los Rumanos, nos dice la princesa,

son más tolerantes que los Occidentales. Ningún Tsigan [sic.] ha estado, en este país, expuesto a los rigores de la ley. También los Domni han alcanzado un logro que parecerá inaudito a aquellos que conocen las costumbres de este pueblo extraño. Ellos han enraizado a una parte de estos nómadas a la tierra de Rumania (p. 105).

En este caso, el mérito de Ghica sería fundamental, porque Alexandru II, en 1836 [sic.], en Valaquia, habría liberado a los Gitanos del Estado y del clero, mientras que Mihail Sturdza no habría seguido este paso en Moldavia hasta 1844. Pero como muestra la tabla 1, si esto es cierto para Moldavia, es falso para Valaquia. La tentativa de hacer de los Ghica los héroes de la emancipación en general reaparece en otra falsedad, cuando Dora d'Istria (p. 85) atribuye a Grigore II Ghica, príncipe de Moldavia, el mérito de haber abolido la servidumbre de la gleba en 1741, acción que todos los historiadores atribuyen sin embargo a Constantin Mavrocordat, cuando era príncipe en Valaquia en 1746 y en Moldavia en 1749 (Papacostea, 1971).

En cuanto a la supuesta no-exposición "a los rigores de la ley", Felix Colson no era de la misma opinión: 
En Valaquia, la ley sacrifica al esclavo, al maestro. En Moldavia, el esclavo tiene el derecho de tener un pequeño peculio. En Valaquia, estos infortunados no gozan de los mismos derechos civiles, y aunque la costumbre admite un contrato entre el amo y el esclavo, por ejemplo, en el caso de un permiso para ir a trabajar a casa de un particular, por medio de un tributo, el cigain no puede denunciar al amo a la justicia hasta que atente contra su vida. La ley moldava es más humana, pero el trato de los amos es también bárbaro. Todos los días, por un plato roto, por un bucle mal rizado, estos desdichados expiran bajo los golpes. Y, sin embargo, no he visto ningún caso de condena de un amo. Y cuando visité las salinas, los presidios donde son enviados los grandes criminales quienes sufren también la pena de muerte, no encontré un solo boyardo (Colson, 1839: 144-145).

Cuando se refiere a los Gitanos en los dos Principados, Dora d'Istria claramente está en apuros: por una parte, no puede ocultar el hecho de que son numerosos $(250.000$ señala en 1859, utilizando datos de Vaillant, que descienden a 80.000 en 1865 , cuando afirma, en cualquier caso, que son más numerosos que en otros lugares); por otra parte, no puede ofrecer un pobre papel a su país en esta tabla comparativa entre el Oriente y el Occidente; y aún más, tiene que confrontar la reputación de liberal que se está construyendo a su medida frente a su sentido de pertenencia a una dinastía que había gobernado dos países cristianos, pero esclavistas, en el curso de los últimos siglos. Por último, se ve obligada a aferrarse a las ramas cuando describe su vida en Rumania sin mencionar a los esclavos, hombres y mujeres, que rondaban a su alrededor mientras vivía en Bucarest. Sencillamente, no habla nunca de los Gitanos esclavos de los boyardos. Y con ello, simplemente, su experiencia es expurgada. Ella engaña de dos maneras: o bien copia a los otros, o bien se evade. Copia incluso cuando finge relatar fragmentos de vida: cita un espectáculo de hombres y mujeres gitanos ofrecido en su honor por la "mujer de un gran boyardo" en Iaşi, haciendo que aparezca incluso el texto en romanés de una canción. Pero toda la página es un plagio, ya que toda la historia es tal cual la relata Augustus De Gérando (1845: 189-190), texto de la canción incluido, una experiencia que el autor francés narraba después de haber vivido en Cluj, en Transilvania ${ }^{4}$, en aquel tiempo bajo la dominación de los Habsburgo.

En este punto, es la construcción del silencio la que nos interesa. ¿Podríamos pensar que la joven princesa Elena no había tenido la experiencia con los Gitanos en su juventud, porque eran poco numerosos en Bucarest? Imposible: según las estadísticas aprobadas por la Asamblea en 1840 (Analele Parlamentare ale Romaniei, 1898), mientras que su tío era un príncipe y ella tenía doce años, se desprende de mis cálculos que en la ciudad de Bucarest y en la provincia de Ilfov que la circunda,

4. Las informaciones de De Gérando también fueron relatadas por Vaillant (1979: 316-318), quien lo citaba. 
en 1839 vivía la mayor concentración de Gitanos en toda Valaquia, más de 12.600 familias, correspondiente al 20\% aproximadamente del total. ¿Podríamos pensar que se trataba sobre todo de esclavos del Estado o de monasterios, lo cual los hacía quizás menos visibles? Imposible: de las 4.609 familias de esclavos Gitanos censados tan solo en la ciudad de Bucarest, más del 90\% pertenecían a los boyardos (véase la Figura 1).

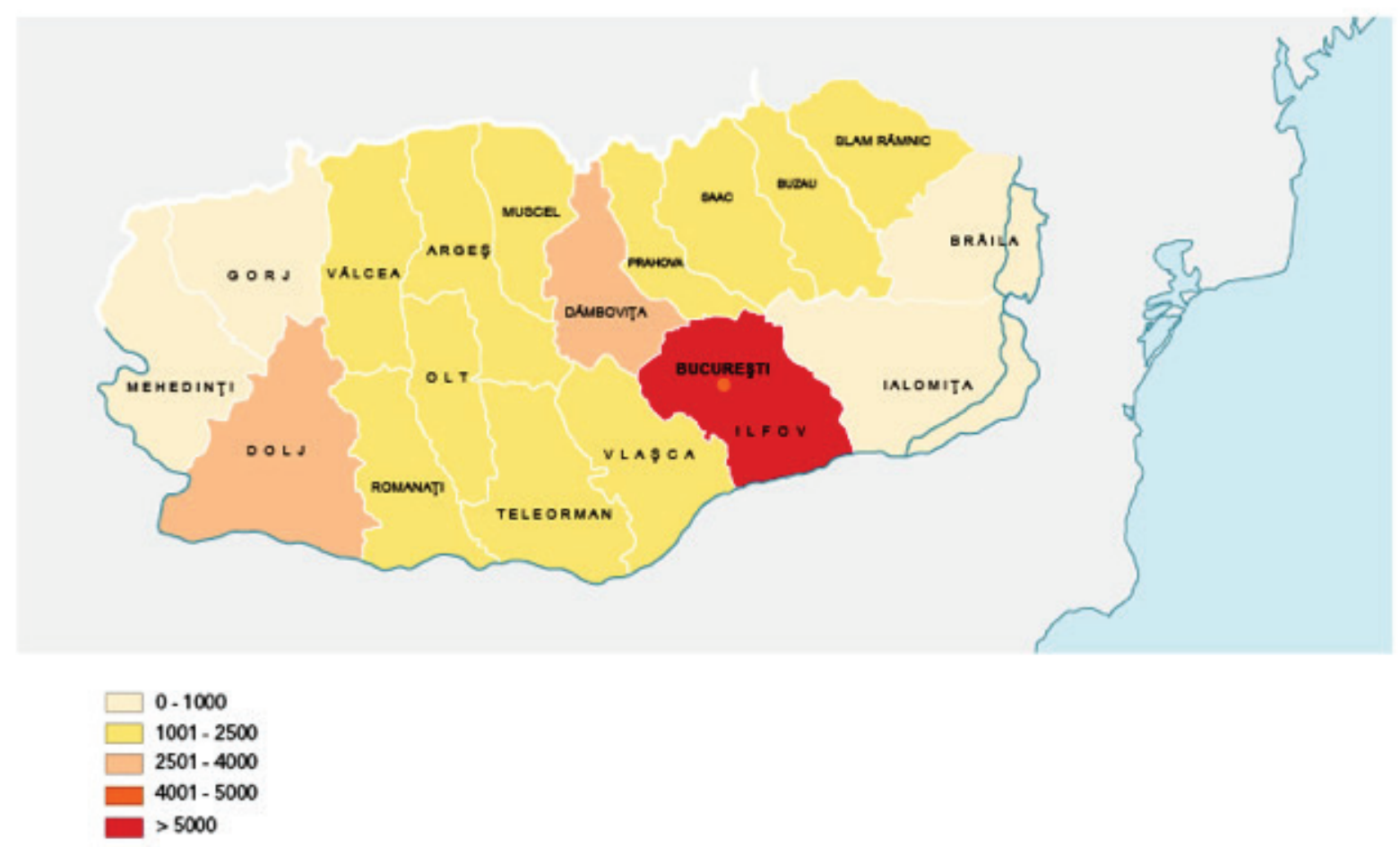

Figura 1 - Distribución de los gitanos esclavos de los boyardos en los distritos de Valaquia, después del censo de 1839 (re-elaboración del autor a partir de datos que figuran en Analele Parlamentare ale Romaniei, 1898). Las cifras se refieren al número de familias.

¿Podríamos pensar que los Ghica no tenían esclavos o eran pocos? Imposible: Vaillant, que conocía muy bien la situación, cita a los Ghica entre las familias que poseen más esclavos en Valaquia, junto con los Bibescu, los Bellu, los Suţu, los Filipescu, los Golescu y los Bălăceanu (1979:357) ${ }^{5}$ ¿ ¿Podríamos pensar que los Gitanos de los boyardos trabajaron principalmente en los campos y no como siervos domésticos? Imposible: Colson señala claramente que la infancia de los pequeños boyardos es "confiada a los esclavos", y que estos niños pasan "la edad que exige toda la atención de una madre en las manos de las niñeras que les hablan sin cesar de fantasmas" (1838:155). Mejor aún, a lo largo de los años, este fenómeno se vuelve casi un lugar común literario y, por lo menos desde Raichevich (1788:254), se atribuye la ignorancia y la mala educación de los boyardos al hecho de que los pequeños son criados por los esclavos gitanos. ¿Podríamos pensar que

5. Según Laurençon, a principios del siglo XIX, los Brâncoveanu poseían ellos solamente de catorce mil a quince mil Gitanos (cf. F. G. L. 1822: 25). Hasta el día de hoy no disponemos de investigaciones precisas sobre las propiedades de esclavos de los boyardos. 
no se hablaba en familia de ello? Difícil de creer: el padre de Elenafue ministro cuando su tío intenta crear granjeros en 1837-39; su hermana Olga se casará con GrigoreSturdza, hijo del príncipe que liberará los Gitanos del Estado y los de la iglesia en 1844 en Moldavia; el príncipe Grigore V Ghica, hijo de uno de sus primos segundos, firmará en 1855 la liberación de los Gitanos de los boyardos siempre en Moldavia, y la hija de este último los emancipará sin reclamar la indemnización prevista por la ley de su padre (Vaillant, 1979:477).

Ante la imposibilidad de desprenderse de ellos, Dora d'Istria suaviza su incómoda presencia transformándolos en servidores exóticos, una artimaña que pone en marcha a través de hábiles expedientes retóricos, la cual se convertirá en un verdadero enfoque historiográfico para los autores rumanos posteriores: comienza diciendo que ellos "solo pueden descender de una tribu de Soudras", la última de las cuatro castas de la India, término que traduce como "siervos" (p. 104); pero sabemos que los śudra no eran siervos; ella continúa citando un estudio realizado por Paul Bataillard en 1849, según el cual, en Rumania, "ya estaban viviendo como siervos a mediados del siglo XIV" (p. 105); sin embargo, Bataillard dice textualmente, "que ya estaban allí, como hoy en día, en estado de esclavitud" (1849: 21) ${ }^{6}$. Ella retoma la distribución de Vaillant (y otros) de los Gitanos de los Principados en tres categorías, vatraschi (vatraşi), laïaschi(lăiăşi) y netotsi (netoţi), pero no dice que los primeros son los esclavos del hogar: ella solo se limita a definirlos como "domésticos, Tsiganisedentarios que realizan las funciones de la domesticidad". Del mismo modo, no dice que los lä̈aschi son artesanos en estado de esclavitud, sino los evoca únicamente en tanto que artesanos nómadas: son los mismos que su padre y su tío intentaron sedentarizar transformándolos en campesinos corbeables. Mientras que los netoţi, los únicos Gitanos que logran escapar del régimen de la esclavitud y de los que no sabemos prácticamente nada, conservaron la etiqueta de "especie de semi-salvajes" que otros ya habían utilizado. Resulta evidente que los vatraşi que debían convivir con ella cuando era joven, no pueden ser presentados como un oprobio de la organización social de su tierra y su familia. Felix Colson señalaba, refiriéndose a los golpes reservados para los esclavos domésticos:

La desgracia está tan fuertemente cincelada en la fisonomía de estos esclavos, que si en un suntuoso banquete usted llega a ver a uno, se le quita el apetito de golpe, y muy a pesar suyo le surgeesta idea: ¡cuántos latigazos en esta cena!

\footnotetext{
6. Paul Bataillard es otro rumanófilo quien recibirá la ciudadanía rumana en 1864. Resaltamos que el trabajo presente de Bataillard, destinado a volver a fechar la llegada de los Gitanos a Rumania, se basa en los documentos que ha recibido de Nicolae Bălcescu, uno de los líderes de la revolución valaca de 1848. Los había descubierto en los archivos del monasterio de Tismana, en Oltenia. Estos documentos siguen siendo a día de hoy las primeras noticias de la presencia de los Gitanos en la región, en la segunda mitad del siglo XIV, y los describen ya como esclavos, como subraya el mismo Bataillard. Los textos han sido publicados recientemente por Petre Petcuţ (2009).
} 
Si usted admira el sanitario de una mujer, se acuerda del dineral que cuesta, y está tentado de huir de horror (1839:147).

Veinte años antes, en un libro que será traducido al francés en 1831, William Wilkinson, cónsul británico en los principados, explicaba a los lectores la violencia que sufrían los Gitanos así como su papel de nodrizas:

Casi en todas las casas los castigos están instituidos para ellos, el más severo de los cuales es el bastinado aplicado a las plantas desnudas de los pies: es ejecutado por otro gitano, bajo la inspección del superintendente, y frecuentemente del dueño o la dueña. Las damas de categoría, no obstante jóvenes y bellas, no muestran la más mínima delicada reticencia en casos similares de autoridad. [ ... ] Los propietarios privados no tienen el poder de muerte sobre ellos; pero ha sucedido alguna vez, que algún infortunado ha sido golpeado hasta la muerte, y ni el gobierno ni lapoblación se enteraron de las circunstancias. Es bajo los cuidados de estos depravados sirvientes, que los hijos de los Boyardos han crecido. Las mujeres de rango más alto que no están habituadas al cuidado de sus hijos, los dejan en manos de las amas de leche gitanas, cuyo modo de vida los expone incesantemente a enfermedades acreditadas como las más perjudiciales para la calidad de su leche, y cuya mala alimentación y sucios hábitos, deben afectar de otras maneras a la constitución de los niños (1820: 174-175).

En un pasaje, Dora d'Istria, censurando las posiciones bajo su punto de vista radicales de la emancipación femenina de su época, criticando el uso del propio término "emancipación" parece re-evocar la vida de Elena Ghica:

La palabra "emancipación" que tantas veces se ha repetido y de la que se han reído tanto, es por desgracia muy oscura. ¿Se entiende que la mujer seguía siendo "esclava" o al menos "sierva"? Si Fourier y las mujeres que han adoptado sus ideas hubieran visto, como yo, "siervos" de cerca, se cuidarían mucho de aplicar una palabra tan fuerte sobre la condición de la mujer occidental. A ella se la trata como "menor", nada es más cierto, y se llama emancipación al acto por el cual un menor de edad es liberado de la tutela. Pero el uso de una palabra que puede tener tres significados diferentes ¿no está plagado de inconvenientes? (1865, vol I: 69).

No obstante, en 1859 la "domesticidad" de los Gitanos se transforma como mucho en un mérito que debe ser perfeccionado con el tiempo:

El príncipe Alexander Ghika ha estado felizmente inspirado al intentar incorporarlos a la vida social. [ ... ] puesto que una parte de este pueblo es predominantemente nómada, $\mathrm{y}$, en todas partes, tienen pavor a la mínima 
sujeción, y se han amoldado, en Rumania, a la domesticidad, ¿por qué no se habituaron con el tiempo a la agricultura quelos moralizaría transformándolos?

Sin embargo, la "domesticidad" puede ser peligrosa: sabemos que en todas partes, dice, retomando las palabras de Esquiros sin citarlo, las mujeres gitanas "tienen una invencible repugnancia a todo hombre que no sea de su raza", pero esto no existe en los Principados, "donde permanecen como domésticas en el seno de relaciones mucho más habituales -a menudo demasiado íntimas- con la población cristiana" (p. 107). Estas son consideraciones que ponen de relieve tanto el deseo de mantener la distancia entre cristianos y "asiáticos", y la crítica de una mujer frente a las relaciones sexuales entre amos y esclavos, apenas esbozada aquí. En cambio, Colson subrayaba:

Las muchachas gitanas en los hogares sirven todas para los caprichos de sus amos; ved la bella moral de esta ley que declara esclavo a todo hijo de esclavo (1839: 147).

Como escribe Antonio d'Alessandri, "Dora d'Istria, quien apoyaba en principio reformas graduales y moderadas, era todavía una aristócrata tanto por nacimiento como por matrimonio" (2007: 155-156) y, podemos añadir, al guardar silencio sobre los esclavos Gitanos, se comportaba como los boyardos que hacían reír de lástima a Felix Colson cuando "hablaban de la abolición de la trata de negros y del progreso de la civilización en Europa" (1838: 147). Dora d'Istria criticaba también la esclavitud de los Negros en América, y sostenía que una doctrina cristiana, al igual que no puede pensar en poder conciliar "la esclavitud con la democracia", como fue el caso en Estados Unidos, igualmente a ella no se le ocurre pensar en mantener la desigualdad entre hombres y mujeres, así que entonó las alabanzas a la Francia libre que había "abierto la ciudad a los negros y mulatos" (1865, vol I: 163-164). Lo que se revela increíble, para nosotros, es que ella era muy consciente de las similitudes entre las situaciones de esclavitud de los Negros y la servidumbre rusa:

Cuarenta millones de rusos viven en la servidumbre [ ... ] ¡qué extraño! El más poderoso Estado autocrático del continente europeo y la gran democracia americana se han puesto de acuerdo hasta el presente para preservar la esclavitud.

Añade en una nota al pie:

Sabemos que el emperador Alejandro II decretó, en principio, la abolición de la servidumbre. En Estados Unidos no es la servidumbre, sino la esclavitud que persiste y parece inalterable (1860, vol II: 143).

Y alaba a la autora de La cabaña del tío Tom: 
Madame Beecher Stowe tiene razón al insistir sobre este punto de vista en su polémica contra los numerosos partidarios con que cuenta la servidumbre entre los republicanos de Estados Unidos. Si ella hubiera estudiado a los siervos de Rusia, este examen le habría proporcionado nuevos argumentos (ibid.: 144).

Al mismo tiempo, criticó las reformas agrarias que tendían a redistribuir la tierra: es justo que los pobres vivan mejor, pero sin quitarle los bienes a los nobles - jescribe varias veces!

Aunque el gobierno obviamente debe ocuparse sin descanso de la abolición de la servidumbre, es también su obligación rigurosa que tome, como hicieron los reyes de la Inglaterra liberal, todas las precauciones necesarias para que esta medida indispensable no se convierta jamás y bajo ningún pretexto en una causa de ruina para las clases superiores. Este deber es aún más ineludible para él [el emperador], la aristocracia rusa personificando, hasta hoy, la civilización nacional. Alexander I está convencido de emancipar a los siervos de las provincias bálticas sin arruinar a la nobleza (ibidem).

Más tarde, emprende la crítica de las reformas agrarias que comenzaron a ver la luz en la Rumania unificada de 1864, y posteriormente la más radical de 1868, precisamente las que introdujeron el capitalismo moderno en el campo (Stahl, 1969). Se ha dicho con razón que "el liberalismo exaltado de la princesa rumana aparece significativamente redimensionado en estas afirmaciones las cuales la ubican, desde el punto de vista social, en posiciones rígidamente conservadoras" (D’Alessandri, 2007: 155): las nacionalidades cristianas de Europa deben tener la misma dignidad entre ellas, y en su seno la mujer debe ser igual al hombre, pero los nobles son nobles y los campesinos son campesinos... No tenemos información precisa, pero sabemos que después de su matrimonio en 1849 con el conde ruso, Elena Mikhailovna Koltzova-Masalskaya había conservado propiedades en Rumania. Ella recibe el usufructo de anualidades hasta su muerte en 1888, lo cual le garantiza la independencia económica de por vida, y en su testamento, cede estas propiedades al municipio de Bucarest (Alessandri, 2007: 233). Sería interesante, aunque no es esencial para nuestra reconstrucción, saber si ella también habría conservado como dote esclavos gitanos y, en caso afirmativo, si, en el momento de la liberación en 1856, los habría emancipado mediante una indemnización o no. Sería interesante también saber si, como tantos hijos de los boyardos, habría lactado de una nodriza gitana.

El hecho es que la educación de Vaillant, gracias a la cual la situación de los Roms rumanos podría haber inspirado a Elena Ghica reflexiones trascendentes sobre los vínculos de dependencia que se establecieron históricamente en Europa, finalmente cayó en saco roto: la historia de familia de la aristocrática Elena bloqueó y censuró las nostalgias de Dora. 


\section{REFERENCIAS BIBLIOGRÁFICAS}

Achim, Viorel (1998) Ţiganii în istoria României. Bucureşti: Editura Enciclopedică.

Achim, Viorel (2004) "The Gypsies in the Romanian Principalities: The Emancipation Laws, 1831-1856". Historical Yearbook, I: 93-120.

Analele Parlamentare ale Romaniei (1898) "Pentru Catagrafia Țiganilor”. Tomul IX, Partea I, Obicinuita Obştanească Adunare a Țĕrei Româneşti, Legislatura II, Sesiunea III (VIII), 1839-1840, Bucuresci: Imprimeria Statului. pp. 1163-1166.

Asséo, Henriette (2008) "Un cosmopolitisme inavouable. Les Bohémiens dans le préromantisme européen”. En Sarga Moussa (coord.): Le Mythe des Bohémiens dans la littérature et les arts en Europe. Paris: L'Harmattan. pp. 83-104.

B. A. [Billecocq, Adolphe] (1847) La principauté de Valachie sous le hospodar Bibesko. Bruxelles: Wouter.

Baldensperger Fernand (1938) "L’entrée pathétique des Tziganes dans les lettres occidentales". Revue de Littérature comparée. XVIII: 587-603.

Bataillard, Paul (1849) Nouvelles recherches sur l'apparition et la dispersion des Bohémiens en Europe. Extrait de la Bibliothèque de l'École des Chartes, $3^{\mathrm{e}}$ série, vol. I. Paris: Franck.

Bengesco, Georges (1895) Bibliographie franco-roumaine du XIXe siècle. Bruxelles: Lacombler.

Bibesco, Georges (1894) Règne de Bibesco. Paris: Plon.

Bodea, Cornelia (1998) "Dora d'Istria through American eyes “. Revista RomânoAmericană, 1: 24-42.

Bordaş, Liviu (2005) "Etnologia e orientalistica nei nuovi stati Italia e Romania: Angelo De Gubernatis, Dora d'Istria e gli studiosi romeni nella seconda metà dell 'XIXe siècle". Annali. Università degli Studi di Napoli "L'Orientale”, 65: 103-119.

Cantemir, Dimitrie (1997) Descrirea Moldovei. Chişinău: Litera [escrito en 1714, edición alemana de 1769].

Colson, Félix (1839) De létat présent et de l'avenir des principautés de Moldavie et de Valachie. Paris: Pougin.

Coquio, Catherine (2011) “Être ou ne pas être 'européen"'. Communication présentée au colloque Tsiganes, nomades, un malentendu européen. Paris, 7 octubre.

D’Alessandri, Antonio (2007) Il pensiero e l'opera di Dora d'Istriafra oriente europeo e Italia, Roma: Gangemi.

De Gérando, Auguste (1845) La Transylvanie et ses habitants. Paris: Au comptoir des Imprimeurs-Unis.

Dibra, Zenepe (2006) "Elena Gjika (Dora d'Istria)", in Francisca De Haan, Krassimira Daskalova, Anna Loutfi (coords.) A Biographical Dictionary of Women's Movements and Feminisms. Central, Eastern, and South Eastern Europe. Budapest: Central European University Press. pp. 158-161. 
Dora d'Istria (1858) La vie monastique dans l'Église orientale, Séconde édition refondue et très-augmentée. Genève: Cherbulier.

Dora d'Istria (1859-1860) Les femmes en Orient. Zurich: Meyer \&Zeller.

Dora d'Istria (1865) Des femmes par une femmes. Paris: Librairie Internationale.

Esquiros, Alphonse (1858) "Les Gyspsies et la vie errante". Revue des Deux Mondes, 15: 721-771.

F. G. L. [F. G. Laurençon] (1822) Nouvelles observations sur la Valachie. Paris: Egron et Ponthieu.

Ferrari, Florencia (2012) 'La 'bonne aventure' par les Calons de São Paulo: entre tromperie, syncrétisme et efficacité”. Brésil(s), 2: 83-106.

Iorga, Nicolae (1918) Histoire des relations entre la France et les Roumains. Paris : Payot. Kogalnitchan, Michel [Kogălniceanu Mihail] (1837a) Histoire de la Valachie, de la Moldavie et des Valaques Transdanubiens. Tome premier. Berlin: Behr.

Kogalnitchan, Michel [Kogălniceanu Mihail] (1837b) Esquisse sur l'histoire, les mours et la langue des Cigains. Berlin: Behr.

Mircea, Ion Radu (1951) “Termenii rob, şerb, şiholopîn documentele slave şi române”, Studii şi cercetari ştiintifice. I (2). pp. 857-873.

Moulier Boutan, Yann (2002) Dalla schiavitù al lavoro salariato. Roma: Manifesto libri.

Okely, Judith (1996) Ownor Other Culture. London: Routledge.

Papacostea, Şerban (1971) "Il regime fanariota in Moldavia e in Valacchia. L'assolutismo asburgico in Transilvania (1711-1820)”. In Andrei Oţetea (coord.), Storia del popolo romeno. Roma: Editori Riuniti. pp. 209-231.

Petcuţ, Petre (2007) "Lapparition des Rroms dans les Prinipautés roumaines et les origines de l'esclavage en Moldavie et Munténie”. Étudestsiganes, n. 29. pp. 38-47.

Petcuţ, Petre (2009) Rromiidin România. Documente. Clij-Napoca: Editura Institutu luipentrustudierea problemi lorminorităţilor naţionale.

Piasere, Leonardo (2004) I romd'Europa. Una storia moderna. Roma e Bari: Laterza.

Piasere, Leonardo (2012) La stirpe di Cus. Storie e costruzioni di unialterità. Roma: CISU.

Raichevich, I. S. [Ignazio Stefano] (1788) Osservazioni storiche, naturali, e politiche in torno la Valachia e Moldavia. Napoli: Raimondo.

Regulamentele organiceale Valahieiş i Moldovei (1944), vol. I, coord. Paul Negulescu, George Alexianu. Bucureşti: Biblioteca Institutului de ŞtinţeAdministrative al României.

Rossi, Luisa (2005) L’altramappa. Esploratrici, viaggiatrici, geografe. Reggio Emilia: Diabasis.

Sauku-Bruci, Merita (2004) Elena Ghika a Girolamo De Rada. Lettere di una principessa, Tirana: Bargjini. 
Stahl, Henri Henri (1969) Anciennes communautés villageoises roumaines. Asservissement et pénétration capitaliste. Paris : CNRS.

Stan, Apostol (1971) Le problème agraire pendant la révolution de 1848 en Valachie. Bucarest: Éditions de l'Académie de la République Socialiste de Roumanie.

Vaillant, Jean Alexandre (1844) La Romanie, ou histoire, langue, littérature, orographie, statistique des peuples de la langued’or, Ardialiens, Vallaques et Moldaves, résuméssous le nom de Romans. Paris: Arthus Bertrand.

Vaillant, Jean Alexandre (1868) Grammaire, dialogues et vocabulaire de la langue des Bohémiensou Cigains. Paris: Maison neuve.

Vaillant, Jean Alexandre (1979 [1857]), Les Rômes. Histoire vraie des vrais bohémiens. Pantin: Les Textes Essentiells.

Wilkinson, William (1820) An account of the principalities of Wallachia and. London: Longman. 